"Following is the Accepted Version of the article

X.W. Ye, P.S. Xi and Marko Nagode (2019)

Extension of REBMIX algorithm to von Mises parametric family for modeling joint distribution of wind speed and direction

published by

Engineering Structures, Volume 183, 15 March 2019, Pages 1134-1145

https://doi.org/10.1016/j.engstruct.2018.08.035.

This article may be used for non-commercial purposes in accordance with Elsevier's Green Open Access policy." 


\title{
Extension of REBMIX algorithm to von Mises parametric family for modeling joint distribution of wind speed and direction
}

\author{
X. W. Ye, P. S. Xi, and Marko Nagode
}

1st author:

Dr. X. W. Ye (Corresponding author)

Associate Professor

Department of Civil Engineering

Zhejiang University

Hangzhou 310058, China

Tel.: +86-571-88208478

Fax: +86-571-88208685

E-mail: cexwye@zju.edu.cn

2nd author:

Mr. P. S. Xi

MSc

Department of Civil Engineering

Zhejiang University

Hangzhou 310058, China

3rd author:

Dr. Marko Nagode

Professor

Faculty of Mechanical Engineering

University of Ljubljana

Askerceva 6, SI-1000 Ljubljana, Slovenia 


\title{
Extension of REBMIX algorithm to von Mises parametric family for modeling joint distribution of wind speed and direction
}

\author{
X. W. Ye ${ }^{1}$, P. S. Xi ${ }^{2}$, and Marko Nagode 3 \\ ${ }^{1), 2)}$ Department of Civil Engineering, Zhejiang University, Hangzhou 310058, China \\ 3) Faculty of Mechanical Engineering, University of Ljubljana, Ljubljana, Slovenia
}

\begin{abstract}
A statistical analysis of the wind speed and wind direction serves as a solid foundation for the wind-induced vibration analysis. The probabilistic modeling of wind speed and direction can effectively characterize the stochastic properties of wind field. The joint distribution model of wind speed and direction involves a circular distribution and has a multimodal characteristic. In this paper, the finite mixture distribution model is introduced and used to represent the joint distribution model that is comprised of the mixture Weibull distributions and von Mises distributions. An extended parameters estimation algorithm for multivariate and multimodal circular distributions is proposed to construct the joint distribution model. The proposed algorithm estimates the component parameters, mixture weight of each component and the number of components successively by an iterative process. The major improvement is accomplished by adding a circular distribution model. The effectiveness of the proposed algorithm is verified with numerical simulations and one-year field monitoring data and compared with the expectation maximization algorithm-based angular-linear approach in terms of the Akaike's information criterion and computing time. The results indicate that the finite mixture model represents the joint distribution model of wind speed and direction well and that the proposed algorithm has a good and time-saving performance in parameter estimation for multivariate and multimodal models.
\end{abstract}

Keywords: Structural Health Monitoring; Wind Field Characteristics; Joint Probability Density Function; Mixture Estimation; REBMIX Algorithm.

\footnotetext{
1 Associate Professor, Department of Civil Engineering, Zhejiang University, Hangzhou 310058, China (corresponding author). E-mail: cexwye@zju.edu.cn

2 MSc, Department of Civil Engineering, Zhejiang University, Hangzhou 310058, China

3 Professor, Faculty of Mechanical Engineering, University of Ljubljana, Ljubljana, Slovenia
} 


\section{Introduction}

For the long-span bridges, the environmental wind load will cause relatively large vibration and deformation with the increase of flexibility and span. As stress cycles would occur under frequent wind load induced by wind speed, critical components of long-span steel bridges may suffer an enormous amount of stress cycles, which will lead to fatigue damage accumulation and finally structural failure may appear without extreme climate condition. In addition to wind speed, wind direction is another important factor when considering the effect of wind load because wind in the along and transverse directions of a bridge will cause a completely different effect (Repetto and Solari, 2004). Due to the wind-induced fatigue damage of long-span steel bridges depending on wind speed and wind direction, the statistical model of field monitoring of wind data near the bridge site will lay a solid foundation for the accurate fatigue evaluation. A suitable model of joint distribution and an efficient parameter estimation method are major challenges for constructing the statistical model of wind speed and direction.

Nowadays, structural health monitoring systems have been installed on structures to measure environmental load and actual structural response of the existing structures. Field monitoring of wind data includes wind speed variable and wind direction variable. For the wind speed variable, the two-parameter Weibull distribution has been commonly used and accepted to model wind speed distribution (Seyit \& Ali, 2009, Bilir et al., 2015). Compared with the literature on wind speed, the literature on wind direction modeling is more limited because the distribution of the wind direction variable is a circular distribution (Erdem \& Shi, 2011). Masseran et al. (2015) used the circular distribution based on nonnegative trigonometric sums as the probability density function of wind direction. Carta et al. (2008a) proposed a finite mixture model comprised of a finite mixture of von Mises distribution to represent the distribution of directional wind speed. Based on the distribution functions of wind speed and direction, there are some approaches to construct the joint distribution model. Feng et al. (2015) constructed the joint distribution of wind speed and direction based on the parameters of the sector-wise Weibull distribution and interpolations between direction sectors. Additionally, the angular-linear distribution models are used to construct joint distributions with specified marginal distributions (Carta et al., 2008b). 
While the models described above are all comprised of one-dimensional marginal distributions that will lead to the deviation between the predictive model and the distribution of observations, very little has been written in the literature about the finite mixture distribution models that are multidimensional.

Many optimization algorithms have been proposed to optimize the joint distribution models by estimating the parameters in models and selecting the optimal joint distribution model, such as the least square method (Carpenter et al., 2014, Carta et al., 2008a), the expectation maximization (EM) algorithm (Ovgor et al., 2012, Calderara et al., 2011) and the genetic algorithm (Holland, 1975). Carta et al. (2008a) used the least squares method to estimate the parameters in a mixture of von Mises distributions. Masseran (2015) separated wind direction data into eight groups based on geographical directions and used Markov chain models to model the distribution. Nasr and Ben (2017) applied three maximum likelihood technics based on Newton Raphson algorithm, Levenberg Marquardt algorithm and Trust Region reflective algorithm to the parameter estimation of the Weibull distribution. Calderara et al. (2011) proposed a new specific EM algorithm for estimating the parameters in a mixture of von Mises distributions. In addition, the EM algorithm has been used widely to estimate parameters for the Weibull distribution (Panteleeva et al., 2015, Moon, 1996). However, the EM algorithm is prone to converge to the local maximum of the likelihood function and has a limitation for the parameter estimation of multidimensional models.

The basic purpose of this paper is to develop an effective and efficient parameter estimation algorithm for the multivariate and multimodal circular distribution. The finite mixture of von Mises distributions is selected as the predictive model of the circular distribution. The unknown parameters in the predictive models are estimated by the extended REBMIX algorithm using an iterative process to assign the component densities one after another to the empirical mixture density. The optimal model will be selected from the models of different component numbers by judging the value of Akaike's information criterion (AIC). This paper is organized as follows: the basic finite mixture model is introduced firstly in Section 2. Moreover, the mixture models of wind direction and the joint mixture models of wind speed and direction are formulated by the mixture of von Mises distributions and the mixture of Weibull-von Mises distributions, 
respectively. Then, Section 3 presents an extended REBMIX algorithm for estimating the unknown parameters in the predictive models and a detailed description of the flow of the algorithm. In Section 4, the extended algorithm is verified by being compared to the simulated data. Meanwhile, its performance of modeling with the field monitoring data, as compared to the performance calculated by the EM algorithm, is presented in Section 5. Finally, the conclusions are listed in Section 6.

\section{Basic Finite Mixture Models}

\subsection{Finite Mixture Distribution Model}

The finite mixture distribution model, in statistics, is a probability distribution of random variables whose populations contain two or more subpopulations. The mixture model represents subpopulations within the global population with no need to identify the subpopulation that each individual observation belongs to. The probability density function of the mixture model can be regarded as a convex combination of other different functions with non-negative weights, which sum to one. The individual probability density functions that make up the mixture distribution are called the mixture components, and the corresponding weights of each component are called the mixture weights (McLachlan and Peel, 2000).

Assuming that we have a $d$ dimensional set of observations $\mathbf{y}=\left[\mathbf{y}_{1}, \mathbf{y}_{2}, \ldots, \mathbf{y}_{d}\right]$ and a dataset of size $n$ with $\mathbf{y}_{i}=\left[\mathrm{y}_{i 1}, \mathrm{y}_{i 2}, \ldots, \mathrm{y}_{i n}\right]$ of continuous or discrete vector observations, the distribution of these observations follows the finite mixture model, and is formulated as

$$
f(\mathbf{y} \mid c, \mathbf{w}, \boldsymbol{\Theta})=\sum_{l=1}^{c} w_{l} f_{l}\left(\mathbf{y} \mid \boldsymbol{\theta}_{l}\right)
$$

with the mixture components $f_{l}\left(\mathbf{y} \mid \boldsymbol{\theta}_{l}\right)$, which is expressed as:

$$
f_{l}\left(\mathbf{y} \mid \boldsymbol{\theta}_{l}\right)=\prod_{i=1}^{d} f_{l}\left(y_{i} \mid \boldsymbol{\theta}_{i l}\right)
$$

where $f(\mathbf{y} \mid c, \mathbf{w}, \boldsymbol{\Theta})$ is the probability density function of the mixture model, $f\left(y \mid \boldsymbol{\theta}_{l}\right)(l=1,2, \ldots, c)$ is a finite set of probability density functions of mixture components, $c$ is the finite number of components, and $w_{l}(l=1,2, \ldots, c)$ is the weight of each corresponding mixture component that 
is greater than zero and sum to one. $\boldsymbol{\Theta}=\left[\boldsymbol{\theta}_{1}, \boldsymbol{\theta}_{2}, \ldots, \boldsymbol{\theta}_{c}\right]$ is the parameter family of all mixture components and $\boldsymbol{\theta}_{l}=\left[\begin{array}{lll}\boldsymbol{\theta}_{1 l}, & \boldsymbol{\theta}_{2 l}, \ldots, \boldsymbol{\theta}_{d l}\end{array}\right]^{\mathrm{T}}$ is the parameter family of distribution functions represented by different dimensional variables in the mixture model.

\subsection{Wind Direction Distribution Model}

Wind direction is usually expressed in an azimuth degree and is measured in degrees clockwise from the north. Thus, the distribution of wind direction is a circular distribution and always ranges from 0 to $2 \pi$. In directional statistics, the von Mises distribution is a continuous distribution on the circle and for the circular observation y, it can be defined as (Mardia and Jupp, 1999)

$$
f_{l}\left(y_{i} \mid \mu_{i l}, \kappa_{i l}\right)=\frac{\exp \left[\kappa_{i l} \cos \left(y_{i}-\mu_{i l}\right)\right]}{2 \pi I_{0}\left(\kappa_{i l}\right)}
$$

where $\mu_{i l}$ represents a measure of the location, which means that the distribution concentrated around $\mu_{i l}$ and $\kappa_{i l}$ is a measure of the concentration analogous to the reciprocal of variance. Additionally, in the denominator of Eq. (3), $I_{0}\left(\kappa_{i l}\right)$ is the modified Bessel function of first kind and order zero. The modified Bessel function of first kind can be expressed as (Abramowitz and Stegun, 1972)

$$
I_{\alpha}\left(\kappa_{i l}\right)=\frac{1}{\pi} \int_{0}^{\pi} e^{\kappa_{i l} \cos \theta} \cos \alpha \theta d \theta-\frac{\sin \alpha \pi}{\pi} \int_{0}^{\infty} e^{-\kappa_{i l} \cosh t-\alpha t} d t
$$

With the von Mises distribution being the most commonly used circular variable distribution, a finite mixture of von Mises distributions is selected to form the wind direction distribution model

$$
f(y \mid c, \mathbf{w}, \boldsymbol{\Theta})=\sum_{l=1}^{c} \frac{w_{l}}{2 \pi I_{0}\left(\kappa_{l}\right)} \exp \left[\kappa_{l} \cos \left(y-\mu_{l}\right)\right]
$$

where $c$ is the number of von Mises components and $w_{l}$ is the weight, where

$$
\sum_{l=1}^{c} w_{l}=1
$$

\subsection{Joint Distribution Model of Wind Speed and Direction}


As for the two-dimensional field monitoring dataset, the joint distribution of wind speed and direction is proposed based on the finite mixture distribution model. Wind speed and direction are assumed to follow the finite mixture model with conditionally independent component densities. The Weibull distribution is applied as the distribution model of wind speed to ensure that the probability of zero and infinity wind speed is zero. The probability density function of the Weibull distribution is

$$
f_{l}\left(y_{i} \mid \alpha_{i l}, \beta_{i l}\right)=\frac{\beta_{i l}}{\alpha_{i l}}\left(\frac{y_{i}}{\alpha_{i l}}\right)^{\beta_{i l}-1} \exp \left[-\left(\frac{y_{i}}{\alpha_{i l}}\right)^{\beta_{i l}}\right]
$$

where $\alpha_{i l}$ represents the scale parameter and $\beta_{i l}$ is the shape parameter of the distribution. The joint distribution model can be presented as

$$
\begin{aligned}
& f(\mathbf{y} \mid c, \mathbf{w}, \boldsymbol{\Theta})=\sum_{l=1}^{c} w_{l} f_{v}\left(y_{1} ; \alpha_{l}, \beta_{l}\right) f_{\theta}\left(y_{2} ; \mu_{l}, \kappa_{l}\right) \\
& =\sum_{l=1}^{c} w_{l} \frac{\beta_{l}}{\alpha_{l}}\left(\frac{y_{1}}{\alpha_{l}}\right)^{\beta_{l}-1} \exp \left[-\left(\frac{y_{1}}{\alpha_{l}}\right)^{\beta_{l}}\right] \frac{\exp \left[\kappa_{l} \cos \left(y_{2}-\mu_{l}\right)\right]}{2 \pi I_{0}\left(\kappa_{l}\right)}
\end{aligned}
$$

where $\mathbf{y}=\left[y_{1}, y_{2}\right]$ stands for the two-dimensional vector variable, i.e. wind speed $y_{1}$ and wind direction $y_{2}$. The estimated parameter $\boldsymbol{\Theta}=\left[\boldsymbol{\alpha}_{l}, \boldsymbol{\beta}_{l}, \boldsymbol{\mu}_{l}, \boldsymbol{\kappa}_{l}\right]$ includes the scale parameter, shape parameter, location parameter and concentrate parameter for each component.

The target of the parameter estimation is to obtain the number of components $c$, corresponding component weights $\mathbf{w}$ and component parameters $\boldsymbol{\theta}_{l}$. The mixture models involve a latent variable, i.e. weights $\mathbf{w}$, unknown parameters $\boldsymbol{\Theta}$ and known data observations. With the latent variables in predictive models, it is usually difficult to obtain optimal solutions by solving the resulting equations of the likelihood function, which is normally a set of interlocking equations. The calculated values of estimated parameters require the values of latent variables and vice versa in this equation set. For estimating the parameters in the mixture model, the main idea in this paper is to assign the component densities one after another to the empirical mixture density for the target joint distribution. In the meantime, the observations belonging to the existing component density will be extracted from the dataset. The number of remained observations in the dataset decreases with the increase of extracting times. When the number of residual observations reaches the lowest value set, they can be distributed into the existing 
components without generating a new component. The proposed parameter estimation framework is described and explained in the next section.

\section{Parameter Estimation Framework}

The REBMIX algorithm for conditionally independent normal, lognormal, Weibull, gamma, binomial, Poisson and Dirac component densities is explained in detail in Nagode (2015), Nagode and Fajdiga (2011a) and Nagode and Fajdiga (2011b). It is depicted in Fig. 1 and is implemented in a freely available R software package rebmix (Nagode, 2018).

\section{Fig. 1. Flowchart of REBMIX}

The paper is focused on the mixtures of von Mises component densities. In the REBMIX algorithm, parameter estimation is largely independent of parametric families. This means that, when it is extended to von Mises parametric family, only the set of equations for rough and enhanced component parameter estimation, first and second moment calculation and the Bayes classification of the unassigned observations have to be derived. The rest remains unchanged.

\subsection{Rough Component Parameter Estimation}

Rough component parameters are used to prevent a component from flowing away from the global mode as at least one component is supposed to be in its vicinity. This yields

$$
f\left(\mathbf{y}=\hat{\mathbf{y}}_{m} \mid \boldsymbol{\theta}_{l}\right)=f_{l m}
$$

where $f_{l m}$ is the global mode, $\hat{\mathbf{y}}_{m}$ is the mean point of the $m$ th bin, $\hat{\mathbf{y}}_{m}=\overline{\mathbf{y}}_{m}$ for the histogram, and $\hat{\mathbf{y}}_{m}=\mathbf{y}_{m}$ for the Parzen window and $k$-nearest neighbours. Eq. (9) is insufficient for the calculation of parameters when the dimension of observations is greater than one. Assuming that components are mutually independent and substituting Eq. (2) into Eq. (9), we obtain

$$
\prod_{i=1}^{d} f\left(y_{i}=\hat{y}_{i m} \mid \boldsymbol{\theta}_{i l}\right)=f_{l m}=\prod_{i=1}^{d} \varepsilon f_{i \mid \hat{i}, l m}
$$

where the required restraints

$$
f\left(y_{i}=\hat{y}_{i m} \mid \boldsymbol{\theta}_{i l}\right)=\varepsilon f_{i \mid \hat{i}, l m}=f_{i \mid \hat{i}, l \mathrm{max}}
$$


can be derived from. For $\varepsilon$ and $f_{i \mid \hat{l}, l m}$ see Nagode (2015).

Another restraint condition is that the predictive component density at the mean point $\hat{\mathbf{y}}_{m}$ attains its maximum value. The method of Lagrange multipliers is used to find the maximum entropy subject to equality constraints. Eq. (11) is first rewritten as

$$
\log \left(f\left(y_{i}=\hat{y}_{i m} \mid \boldsymbol{\theta}_{i l}\right) / f_{i \mid \hat{i} . l \max }\right)=0
$$

Next, Lagrange multiplier $\lambda_{i l}$ is introduced and the Lagrange function defined by

$$
\begin{aligned}
\Lambda\left(\boldsymbol{\theta}_{i l}, \lambda_{i l}\right)= & -\int_{-\infty}^{+\infty} f\left(y_{i} \mid \boldsymbol{\theta}_{i l}\right) \log \left(f\left(y_{i} \mid \boldsymbol{\theta}_{i l}\right)\right) d y_{i} \\
& +\lambda_{i l} \log \left(f\left(y_{i}=\hat{y}_{i m} \mid \boldsymbol{\theta}_{i l}\right) / f_{i \mid \hat{i} . l \text { max }}\right)
\end{aligned}
$$

provides a strategy for entropy maximization. The rough component von Mises parameters are then a solution of

$$
\nabla_{\boldsymbol{\theta}_{i l}, \lambda_{i l}} \Lambda\left(\boldsymbol{\theta}_{i l, \lambda_{i l}}\right)=0
$$

where index $i=1, \ldots, d$ and index $\hat{\imath}=1, \ldots, i-1, i+1, \ldots, d, \hat{y}_{i m}=\bar{y}_{i m}$ for the histogram and $\hat{y}_{i m}=$ $y_{i m}$ for the Parzen window and $k$ nearest neighbours. If Eq. (3) is put in Eq. (13), we get

$$
\begin{aligned}
\Lambda= & -\kappa_{i l} \frac{I_{1}\left(\kappa_{i l}\right)}{I_{0}\left(\kappa_{i l}\right)}+\log \left(2 \pi I_{0}\left(\kappa_{i l}\right)\right)\left(1-\lambda_{i l}\right) \\
& +\lambda_{i l} \kappa_{i l} \cos \left(\hat{y}_{i m}-\mu_{i l}\right)-\lambda_{i l} \log \left(f_{i \mid \hat{i} . l \max }\right)
\end{aligned}
$$

At this point, we treat $\mu_{i l}$ and $\kappa_{i l}$ as the estimated parameters and insert Eq. (15) into Eq. (14), and then the gradients of the Lagrange function are obtained as follows

$$
\frac{\partial \Lambda}{\partial \mu_{i l}}=\lambda_{i l} \kappa_{i l} \sin \left(\hat{y}_{i m}-\mu_{i l}\right)=0
$$

and

$$
\frac{\partial \Lambda}{\partial \lambda_{i l}}=\kappa_{i l}-\log \left(I_{0}\left(\kappa_{i l}\right)\right)-\log \left(2 \pi f_{i \mid \hat{i} . l \max }\right)=0
$$

namely

$$
f\left(\mu_{i l}\right)=\lambda_{i l} \kappa_{i l} \sin \left(\hat{y}_{i m}-\mu_{i l}\right)=0
$$

and 


$$
f\left(\kappa_{i l}\right)=\kappa_{i l}-\log \left(I_{0}\left(\kappa_{i l}\right)\right)-\log \left(2 \pi f_{i \mid \hat{i} . l \max }\right)=0
$$

where it follows that rough measure of location parameters $\mu_{i l}=\hat{y}_{i m}$ satisfy Eq. (18) and the derivative of Eq. (19) is defined as

$$
f^{\prime}\left(\kappa_{i l}\right)=1-\frac{I_{1}\left(\kappa_{i l}\right)}{I_{0}\left(\kappa_{i l}\right)}
$$

Hence the rough measure of concentration parameters $\kappa_{i l}$ can be derived from Eq. (20) by a root-finding algorithm, e.g., the Newton-Raphson method.

\subsection{Enhanced Component Parameter Estimation}

To assess enhanced component parameters for the histogram, the log-likelihood

$$
\log L\left(\hat{y}_{i 1}, \ldots \hat{y}_{i v} \mid \boldsymbol{\theta}_{i l}\right)=\sum_{j=1}^{v} k_{l j} \log \left(f\left(\hat{y}_{i j} \mid \boldsymbol{\theta}_{i l}\right)\right)
$$

is maximized. Unknown parameters $\boldsymbol{\theta}_{i l}$ are derived for the histogram, where $v$ and $k_{l j}$ stand for the number of bins and the frequencies of the $j$ th component, respectively. If index $v$ is replaced by $n$ in Eq. (21), the equations of this section hold also for the Parzen window or $k$ nearest neighbour.

Let Eq. (3) be first put in Eq. (21), which yields

$$
\log L=\sum_{j=1}^{v} k_{l j} \kappa_{i l} \cos \left(\hat{y}_{i j}-\mu_{i l}\right)-n \log (2 \pi)-n \log \left(I_{0}\left(\kappa_{i l}\right)\right)
$$

From the first partial derivative of the log-likelihood function with respect to $\mu_{i l}$ defined as

$$
\frac{\partial \log L}{\partial \mu_{i l}}=\kappa_{i l} \sum_{j=1}^{v} k_{l j} \sin \left(\hat{y}_{i j}-\mu_{i l}\right)=0
$$

it follows that, by applying the sine trigonometric identity, the enhanced measure of location can be expressed as

$$
\mu_{i l}=\arctan \left(\frac{\operatorname{Im}\left(z_{i l}\right)}{\operatorname{Re}\left(z_{i l}\right)}\right)
$$

where the real and imaginary part of a complex number

$$
z_{i l}=\operatorname{Re}\left(z_{i l}\right)+i \operatorname{Im}\left(z_{i l}\right)
$$


are given by

$$
\operatorname{Re}\left(z_{i l}\right)=\frac{1}{n} \sum_{j=1}^{v} k_{l j} \cos \left(\hat{y}_{i j}\right)
$$

and

$$
\operatorname{Im}\left(z_{i l}\right)=\frac{1}{n} \sum_{j=1}^{v} k_{l j} \sin \left(\hat{y}_{i j}\right)
$$

Similarly, the first partial derivative with respect to $\kappa_{i l}$ is defined as

$$
\frac{\partial \log L}{\partial \kappa_{i l}}=\sum_{j=1}^{v} k_{l j} \cos \left(\hat{y}_{i j}-\mu_{i l}\right)-n \frac{I_{1}\left(\kappa_{i l}\right)}{I_{0}\left(\kappa_{i l}\right)}=0
$$

By applying the cosine trigonometric identity, we get

$$
\frac{I_{1}\left(\kappa_{i l}\right)}{I_{0}\left(\kappa_{i l}\right)}=\operatorname{Re}\left(z_{i l}\right) \cos \left(\mu_{i l}\right)+\operatorname{Im}\left(z_{i l}\right) \sin \left(\mu_{i l}\right)=R
$$

where $R$ stands for the absolute value of the complex number

$$
R=\sqrt{\operatorname{Re}\left(z_{i l}\right)^{2}+\operatorname{Im}\left(z_{i l}\right)^{2}}
$$

with the real and imaginary part of the complex number expressed as

$$
\operatorname{Re}\left(z_{i l}\right)=R \cos \left(\mu_{i l}\right)
$$

and

$$
\operatorname{Im}\left(z_{i l}\right)=R \sin \left(\mu_{i l}\right)
$$

Thus, Eq. (29) finally yields

$$
f\left(\kappa_{i l}\right)=I_{1}\left(\kappa_{i l}\right)-R I_{0}\left(\kappa_{i l}\right)=0
$$

and

$$
f^{\prime}\left(\kappa_{i l}\right)=I_{0}\left(\kappa_{i l}\right)-\left(R+\frac{1}{\kappa_{i l}}\right) I_{1}\left(\kappa_{i l}\right)
$$

Therefore, the enhanced measure of concentration $\kappa_{i l}$ can also be regained by a root-finding algorithm, e.g., the Newton-Raphson method. 


\subsection{First and Second Moment Calculation}

The first and second moments are required in the proposed REBMIX algorithm for the Bayes classification of the unassigned components (Nagode, 2015). However, for circular distributions, it turns out that it is more favorable to calculate the real and imaginary parts of the complex number $z_{i l}$ instead of calculating the first and second moment. The real and imaginary parts of the complex number are defined by Eq. (31) and (32).

The reasons become clear later. In Eqs. (31) and (32), parameters $\mu_{i l}$ and $\kappa_{i l}$ are those from the enhanced component parameter estimation.

\subsection{Bayes Classification of Unassigned Observations}

When the assignment of a new component stops, the unassigned observations join the existing components and do not form new ones according to the Bayes decision rule (Duda and Hart, 1973)

$$
l=\underset{l}{\arg \max } w_{l} f\left(\mathbf{y}_{j} \mid \boldsymbol{\theta}_{l}\right)
$$

Once the component membership $l$ for unassigned observations $k_{i l}$ is known, the corresponding component weight

$$
w_{l}=w_{l}+\frac{k_{l j}}{n}
$$

real part

$$
\operatorname{Re}\left(z_{i l}\right)=\operatorname{Re}\left(z_{i l}\right)+\frac{k_{l j}\left(\cos \left(y_{i j}\right)-\operatorname{Re}\left(z_{i l}\right)\right)}{n w_{l}}
$$

and the imaginary part of the complex number

$$
\operatorname{Im}\left(z_{i l}\right)=\operatorname{Im}\left(z_{i l}\right)+\frac{k_{l j}\left(\sin \left(y_{i j}\right)-\operatorname{Im}\left(z_{i l}\right)\right)}{n w_{l}}
$$

are recalculated. Eqs. (36) to (38) are repeated for $j$ ranging from 1 to $v$ for the histogram or to $n$ for the Parzen window or $k$ nearest neighbours. The final measure of location is then given by Eq. (24), whereas the final measure of concentration is obtained only by solving Eq. (29). 


\subsection{Selection of Optimal Model}

To find the number of bins or nearest neighbours resulting in the lowest value of the information criterion, loop 4 in Fig. 1 runs for the range of bins. The algorithm presumes only one component initially and calculates the information criterion for a mixture with that component. Onwards the number of components increases gradually and the information criterion is calculated for the corresponding mixtures. Loops 3 and 2 stop when number of components $c \geq c_{\max }$ or $c \geq v$ or $c \geq$ $k$. Here, $c_{\max }$ stands for the maximum number of components.

The AIC information criterion is used throughout the paper as a criterion for model selection among a finite set of models. It is defined as

$$
\mathrm{AIC}=-2 \log L(c, \mathbf{w}, \boldsymbol{\Theta})+2 M
$$

where $M$ is the number of unknown parameters in the mixture model which equals the number of parameters in probability density functions of mixture components multiplied by $c$ plus $c-1$. The log-likelihood function for the binned observations is given by

$$
\log L(c, \mathbf{w}, \boldsymbol{\Theta})=\sum_{j=1}^{v} k_{j} \log f\left(\mathbf{y}_{j} \mid c, \mathbf{w}, \boldsymbol{\Theta}\right)
$$

where $k_{j}$ represents the frequency of observations falling into the $j$ th bin. For the Parzen window or $k$ nearest neighbours

$$
\log L(c, \mathbf{w}, \boldsymbol{\Theta})=\sum_{j=1}^{v} \log f\left(\mathbf{y}_{j} \mid c, \mathbf{w}, \boldsymbol{\Theta}\right)
$$

As shown in Eq. (39), the AIC evaluates the goodness of fit of models as assessed by the likelihood function. Given a set of models for measured data, the optimal model is the one with the maximum likelihood function value and minimum AIC value.

Loop 1 in Fig. 1 splits the dataset into two clusters, the one corresponding to the currently observed component and the residue. The former is used to estimate the component weight and rough component parameters. The latter is split into two clusters repeatedly until $c \geq c_{\max }$ or $c \geq v$ or $c \geq k$. When the total of positive relative deviations

$$
D=\sum_{j=1}^{v}\left\langle\frac{k_{j}}{n}-f\left(\overline{\mathbf{y}}_{j} \mid c, \mathbf{w}, \boldsymbol{\Theta}\right) V_{j}\right\rangle
$$


is less or equal than $D_{\min } / w_{l}$ or counter $I_{2}$ of the loop 1 attains its maximum $I_{\max }$ or $n_{l} \leq 1$, the enhanced component parameters are estimated and loop 1 stops. Constant $D_{\min }$ is optimized by the algorithm. If $D_{\min }=1$, then $c=1$ and if $0<D_{\min }<1$, then $\mathrm{c} \geq 1$. Here $n_{l}$ stands for the number of observations in the $l$ th component. For the Parzen window or $k$ nearest neighbors algorithm

$$
D=\sum_{j=1}^{n}\left\langle\frac{1}{n}-\frac{f\left(\mathbf{y}_{j} \mid c, \mathbf{w}, \boldsymbol{\Theta}\right) V_{j}}{k_{j}}\right\rangle
$$

where $\langle x>=x$ if $x>0$, and $<x>=0$ if $x<0$. The optimal model with the lowest AIC and D values is selected from all models.

Further details on the REBMIX algorithm can be found in Nagode (2015), Nagode and Fajdiga (2011a) and Nagode and Fajdiga (2011b).

\section{Numerical Examples}

To verify the applicability and effectiveness of the proposed REBMIX algorithm in parameters estimation of finite mixture circular distribution models, two examples of numerical simulations have been tested here. Herein, the proposed algorithm is applied to estimate the parameters of mixture models without being compared to the EM algorithm. In example 1, the datasets of size $n$ $=10000$ generated from the one, two and three components mixture of von Mises distributions are applied for univariate modeling of the multimodal distributions. In example 2, the two-dimensional datasets of size $n=1000000$ generated from the one, two and three components bivariate mixture of Weibull-von Mises distributions have been employed for multivariate modeling of the multimodal distributions.

\subsection{Application Example for Univariate Modeling}

In example 1, three cases with one, two and three components mixture of von Mises distributions have been investigated. The datasets of size $n=10000$ are generated by Monte Carlo simulation, e.g. the slice sampling algorithm, from the target distributions for case 1, case 2 and case 3 , respectively, and the parameter values for each case are shown in Table 1.

The proposed REBMIX algorithm is applied to estimate the parameters of the predictive 
models. In REBMIX algorithm, the preprocessing is set to histogram, the maximum number of components to 10 and the information criterion to $\mathrm{AIC}$. The number of bins $\mathrm{K}$ is set to range from 20 to 40 . The acceleration rate is set to 0.1 and $D_{\min }$ to 0.25 . The results of parameter estimation of cases 1, 2 and 3 are shown in Figs 2 to 4.

Fig. 2. Results of parameter estimation by REBMIX algorithm for case 1

Fig. 3. Results of parameter estimation by REBMIX algorithm for case 2

Fig. 4. Results of parameter estimation by REBMIX algorithm for case 3

In the numerical examples for univariate mixtures, three indexes have been considered to determine an optimal model, i.e. the total of positive relative deviations and the information criterion values of different components and different bin numbers. For Section 4.1, the following six parts are shown in Figs. 2 to 4:

1) $y_{1}-f\left(y_{1}\right)$ : the curve is the probability density function of the predictive von Mises distribution and the points are the probability densities of the generated dataset in each bin, respectively;

2) $c-\mathrm{AIC}, c-\log \mathrm{L}, c-\mathrm{D}$ and $c$ - Time: the points on the line chart represent a list of vectors containing information criteria values, log-likelihood values, positive relative deviations and computing time depending on numbers of components $c$, respectively. The optimal model always has the lowest AIC and D values;

3) K - AIC: the points on the line chart represent a list of vectors containing information criteria values depending on numbers of bins $v$. The optimal number of bins always has the lowest AIC value.

As shown in Fig. 2, it is seen that AIC values increase with the number of components, which means the fitting performance of multicomponent model gets worse rather than better. This happens because there are many unassigned observations after extracting the first component observations from the dataset and these observations cannot be efficiently assigned into the newly added component. With the increase of the number of estimated parameters in the models, the AIC values increase and the D values decrease. It can be found from part (5) in Fig. 2 
that the AIC values reach the minimum at the number of bins equaling 36 and the predictive probability density function could well match the density distribution of simulated observations. The computing time increases proportionally with the number of $c_{\max }$ in less than 15 seconds, which means that the computing time of each component is equal. The estimated values of parameters for optimal models are listed in Table 1 and are compared with the true values.

As shown in Fig. 3, the AIC values rapidly decrease to the minimum value and start to get smooth at two components. According to the Eq. (63) of AIC, it means that the log-likelihood function for the observations has reached its maximum at two components and will not reach better results with more components. In order to avoid overfitting, the penalty term, i.e. the number of components, has been considered. Thus, the optimal model in case 2 is the two component mixture of the von Mises distributions. In the same way, the optimal model in case 3 is the three component mixture of von Mises distributions based on AIC values depending on the numbers of components. From the predictive probability density function in cases 2 and 3 it is obvious that the predictive functions match the distribution of observations well. The number of bins is set to 40 for case 2, and to 39 for case 3 according to the AIC values. Comparing the computing time of three cases, we can find out that the computing time for multimodal observations is similar to unimodal observations. Moreover, the comparison between true values and predictive values of parameters are listed in Table 1. It is seen that the REBMIX algorithm could efficiently estimate the parameters in the complex distributions.

Table 1. True values and predictive values of parameters in example 1

The information criteria are used to measure the fit performance of models in order to choose the optimal number of components, the optimal number of bins and evaluate the fitting degree of the estimated models.

\subsection{Application Example for Multivariate Modeling}

In example 2, the other three cases with a one, two and three component mixture of Weibull-von Mises distributions have been investigated. To ensure the two dimensional simulated data follow mixture of Weibull-von Mises distributions, the datasets of size $n=1000000$ are generated by 
Monte Carlo simulation, e.g., the slice sampling algorithm, from the target distributions for case 4, case 5 and case 6 , respectively, and the parameter values for each case are shown in Table 2 . The REBMIX algorithm is also employed to estimate the parameters in the models and the initial setting of REBMIX algorithm is identical to that in Section 4.1. For Section 4.2, the following eight parts will be shown in the results:

1) $y_{1}-y_{2}$ : the colored circles are contour line of the joint probability density function of predictive mixture of Weibull-von Mises distributions and the points are the probability densities of the generated dataset in each bin, respectively. The same color stands for the same probability density;

2) $y_{1}-f\left(y_{1}\right)$ and $y_{2}-f\left(y_{2}\right)$ : the curve is the marginal probability density function of the Weibull distribution and von Mises distribution in the mixture distribution, respectively, and the points represent the marginal probability densities of the generated dataset in each bin;

3) $c$ - AIC, $c-\operatorname{logL}, c-\mathrm{D}, \mathrm{K}-\mathrm{AIC}$ and $c$ - Time: as indicated in Section 4.1;

For case 4, the one component Weibull-von Mises mixture modeling, the AIC value reaches its minimum at one component and gradually increases. The reason for this phenomenon in bivariate modeling is similar to that in univariate modeling (see Section 4.1). As shown in Fig. 5, the joint probability density function and two marginal probability density functions show good performance. The estimated parameters are listed in Table. 2 .

\section{Fig. 5. Results of parameter estimation by REBMIX algorithm for case 4}

\section{Table 2. True values and predictive values of parameters in example 2}

For case 5, the AIC and D values reach its minimum and start to flatten from two components. It is reasonable to choose the two component Weibull-von Mises mixture model as the optimal model. For case 6, the three component Weibull-von Mises mixture model is regarded as the optimal model according to the AIC values. From the joint probability density function and marginal probability density function of the optimal models in Figs. 6 and 7, it can be stated that the predictive models fit the generated observations well. All estimated values of parameters for optimal models are listed in Table. 2. Tables 1 and 2 show that the estimated values of parameters 
are near the true values of parameters, which can prove that the REBMIX algorithm can estimate parameters of finite mixture circular distribution models efficiently.

Fig. 6. Results of parameter estimation by REBMIX algorithm for case 5

Fig. 7. Results of parameter estimation by REBMIX algorithm for case 6

\section{Field Monitoring Application}

In order to demonstrate the effectiveness of the REBMIX algorithm in univariate and bivariate circular distribution modeling, one-year wind field monitoring data, including a total of 52560 mean wind speed and wind direction data during ten minutes, measured by the ultrasonic anemometer in structural health monitoring system is employed for the construction of the distribution of wind direction and the joint distribution of wind speed and direction by the proposed algorithm and comparison methods, EM algorithm and EM algorithm-based angular-linear modeling approach.

\subsection{Modeling the Distribution of Wind Direction}

For modeling the distribution of wind direction, both the proposed algorithm and the EM algorithm are applied. In the REBMIX algorithm, the preprocessing is set to histogram, the maximum number of components to 20 to ensure sufficient components and the information criterion to AIC. The number of bins $\mathrm{K}$ is set to range from 20 to 40 . In the EM algorithm, the iterative 5000 times or the relative change of parameter values of less than 0.001 as the termination conditions.

The field monitoring data of wind direction ranges from 0 to $2 \pi$, and is divided into 37 bins with an equal width according to the lowest AIC value, as shown in Fig. 8. Based on the corresponding probability density of each bin, the parameters in the mixture von Mises model have been estimated. What's more, the EM algorithm is used to calculate the parameters in the mixture model with different component numbers. Fig. 9 shows the values of AIC with different numbers of components of the von Mises mixture models. It can be found from Fig. 9 that, for the REBMIX algorithm, the AIC values become stable and receive its minimum from seventeen 
components, and the AIC values of the models estimated by the EM algorithm start flattening from fifteen components. Thus, the seventeen component von Mises mixture model with the AIC value of 132790 based on the REBMIX algorithm and the fifteen component von Mises mixture model with the AIC value of 132691 are selected as the optimal mixture models, respectively.

Fig. 8. AIC values with different numbers of bins

Fig. 9. AIC values of models estimated by REBMIX and EM algorithm

According to the AIC value, the optimal models of wind direction are shown in Fig. 10. It is seen that the red line is the probability density function based on the REBMIX algorithm and it matches almost all points of the histogram. However, the probability density function based on the EM algorithm in blue deviates from the points of the histogram. The REBMIX algorithm could estimate the parameters of mixture models that express the probability density distribution of the histogram from preprocessing. Due to the REBMIX algorithm based on the histogram, the AIC value of the optimal model estimated by REBMIX algorithm is slightly higher than the AIC value estimated by the EM algorithm. Fig. 11 shows the computing time of two algorithms. The REBMIX algorithm results in the optimal seventeen component mixture model in $122.5 \mathrm{~s}$, whereas the EM algorithm results in the optimal fifteen component mixture model in $3936.5 \mathrm{~s}$. Thus, the REBMIX algorithm is time efficient and robust.

Fig. 10. Optimal models by REBMIX and EM

Fig. 11. Computing time by REBMIX and EM

\subsection{Modeling Joint Distribution of Wind Speed and Direction}

For modeling the joint mixture distribution of wind speed and direction, the EM algorithm-based angular-linear modeling approach is applied to be compared with the proposed REBMIX algorithm. In the REBMIX algorithm, the pre-processing is set to histogram, the maximum number of components to 20 and the information criterion to AIC. The number of bins $\mathrm{K}$ is set to range from 20 to 50. For the EM algorithm-based angular-linear modeling approach, the first step is to construct the univariate marginal distribution of wind speed and direction by the EM algorithm, then the circular variate is calculated and the distribution of the circular variable is 
constructed. After that, the joint mixture model of wind speed and direction can be obtained by a combination of these three marginal distributions.

Fig. 12 shows the values of AIC with different numbers of bins and the AIC value achieve the minimum value when the number of bins equals 31 . Thus, the observations are divided into 31 bins in the preprocessing and the corresponding probability densities are calculated. The AIC values of predictive models estimated by the REBMIX algorithm are shown in Fig. 13. It is seen that the AIC values begin to become steady from sixteen components and achieve the minimum values at seventeen components. Therefore, the seventeen component mixture of Weibull-von Mises distributions with the AIC value of 264616 constructed by the REBMIX algorithm in 474.6s is regarded as the optimal mixture model, as shown in Fig. 14.

\section{$\underline{\text { Fig. 12. AIC values with different numbers of bins }}$}

\section{Fig. 13. AIC values of models estimated by REBMIX algorithm}

\section{Fig. 14. Optimal model by REBMIX algorithm}

For the EM algorithm-based angular-linear modeling approach, the marginal probability density functions of wind speed, wind direction and circular variable are calculated firstly by the EM algorithm. According to the AIC values, the AIC values of the wind speed model and the circular variable model stabilize from seven components and five components, respectively. Thus, the mixture of seven Weibull distributions is chosen as the wind speed model and the mixture of five von Mises distributions is selected as the circular variable model. Fig. 15 illustrates the optimal models of wind speed and circular variable. Then, the joint mixture model of wind speed and direction is constructed by combining the obtained marginal probability density function of wind speed, direction and circular variable. The joint mixture model of wind speed and direction is shown in Fig. 16. The EM algorithm-based angular-linear modeling approach results in the joint mixture model with the AIC value of 279145 in 11022.4s. Comparing the AIC value and computing time between two approaches, it is obvious that the proposed REBMIX algorithm has a better and time-saving performance in parameter estimation for multivariate and multimodal models as compared to the EM algorithm. 
Fig. 15. Optimal model of wind speed and circular variable

Fig. 16. Optimal model by EM algorithm-based angular-linear modeling approach

\section{Conclusions}

In this paper, an improved parameter estimation algorithm for estimating unknown parameters in multivariate and multimodal circular distributions is presented in detail. The predictive models select the finite mixture of von Mises distributions to characterize the distributions of circular variables. The unknown parameters for the mixture components are estimated through the proposed REBMIX algorithm. The estimated model with the lowest AIC value is selected as the optimal mixture model from the models of all component numbers. Numerical simulations and field monitoring data are used to verify the effectiveness and validity of the proposed algorithm by comparing it with the EM algorithm in accordance with the value of AIC and computing time.

Based on the comparative analysis of the modeling of numerical simulation and field monitoring data by the proposed REBMIX algorithm and EM algorithm, the following conclusions are drawn: (i) the distribution of circular observations, i.e. wind direction data, can be effectively represented by the finite mixture of von Mises distributions; (ii) the proposed REBMIX algorithm can accomplish the parameter estimation of the predictive models; (iii) the joint mixture distribution model of wind speed and wind direction can match the multimodal distribution of field monitoring data; and (iv) the comparison results between the proposed algorithm and the EM-based modeling approach in the modeling of monitoring wind data indicates that the proposed algorithm has a better performance with less computing time.

\section{Acknowledgments}

The work described in this paper was jointly supported by the National Science Foundation of China (Grant No. 51778574), the Fundamental Research Funds for the Central Universities of China (Grant No. 2017QNA4024), and the Slovenian program Nr. P2-0182 entitled Development evaluation financed by the Slovenian Ministry of Education, Science and Sport. 


\section{References}

Abramowitz, M. \& Stegun, I. A. (1972), Handbook of mathematical functions with formulas, Dover Publications, New York, USA.

Bilir, L., İmir, M., Devrim, Y. \& Albostan, A. (2015), An investigation on wind energy potential and small scale wind turbine performance at İncek region - Ankara, Turkey, Energy Conversion \& Management, 103, 910-923.

Calderara S., Prati A. \& Cucchiara R. (2011), Mixtures of von Mises Distributions for People Trajectory Shape Analysis, Transactions on Circuits \& Systems for Video Technology, 21(4), 457-471.

Carpenter, H. W., Reid, R. G. \& Paskaramoorthy, R. (2014), Measurement of the Distribution of Residual Stresses in Layered Thick-Walled Gfrp Pipes, Experimental Mechanics, 54(9), $1627-1638$.

Carta, J. A., Bueno, C. \& Ramírez, P. (2008a), Statistical modelling of directional wind speeds using mixtures of von Mises distributions: Case study, Energy Conversion and Management, 49(5), 897-907.

Carta, J. A., Ramírez, P. \& Bueno, C. (2008b), A joint probability density function of wind speed and direction for wind energy analysis, Energy Conversion \& Management, 49(6), 1309-1320.

Duda, R. O. \& Hart, P. E. (1973), Pattern Classification and Scene Analysis, John Wiley \& Sons, New York, USA.

Erdem E. \& Shi J. (2011), ARMA based approaches for forecasting the tuple of wind speed and direction, Applied Energy, 88, 1405-1414.

Feng, J., Shen, W. Z. \& Sciubba, E. (2015), Modelling wind for wind farm layout optimization using joint distribution of wind speed and wind direction, Energies, 8(4), 3075-3092.

Holland, J. H. (1975), Adaptation in natural and artificial system, The University of Michigan Press, Ann Arbor, USA. 
Mardia, K. V. \& Jupp, P. E. (1999), Directional Statistics, Wiley, New York, USA.

Masseran, N., Razali, A. M., Ibrahim, K., Zaharim, A. \& Sopian, K. (2015), Fitting a circular distribution based on nonnegative trigonometric sums for wind direction in Malaysia, Ism International Statistical Conference with Applications in Sciences \& Engineering, 1643, 305-311.

Masseran N. (2015), Markov chain model for the stochastic behaviors of wind-direction data, Energy Conversion and Management, 92, 266-274.

McLachlan, G. J. \& Peel, D. (2000), Finite Mixture Models, Wiley, New York, USA.

Moon, T. K. (1997), The expectation-maximization algorithm, IEEE Signal Processing Magazine, 13(6), 47-60.

Nagode, M. (2018), rebmix: finite mixture modeling, clustering \& classification, R package version 2.10.2, http://CRAN.R-project.org/package=rebmix.

Nagode, M. (2015), Finite mixture modeling via REBMIX, Journal of Algorithms and Optimization, 3(2), 14-28.

Nagode, M. \& Fajdiga, M. (2011a), The REBMIX algorithm and the univariate finite mixture estimation, Communications in Statistics - Theory and Methods, 40(5), 876-892.

Nagode, M. \& Fajdiga, M. (2011b), The REBMIX algorithm for the multivariate finite mixture estimation, Communications in Statistics - Theory and Methods, 40(11), 2022-2034.

Nasr, A., Gasmi, S. \& Ben Hmida, F. (2017), Parameter estimation of the flexible weibull distribution for type I censored samples, Journal of Applied Statistics, 44(14), 2499-2512.

Ovgor, B., Lee, S.-K. \& Lee, S. (2012), A Method of Micrositing of Wind Turbine on Building Roof-Top by Using Joint Distribution of Wind Speed and Direction, and Computational Fluid Dynamics, Journal of Mechanical Science and Technology, 26(12), 3981-3988.

Panteleeva, O. V., González, E. G., Huerta, H. V. \& Alva, J. A. V. (2015), Identifiability and comparison of estimation methods on Weibull mixture models. Communications in Statistics - Simulation and Computation, 44(7), 1879-1900. 
Pearson, K. (1998), Contributions to the mathematical theory of evolution, Philosophical Transactions of the Royal Society of London, 186(4), 343-414.

Repetto, M. P. \&Solari, G. (2004), Directional wind-induced fatigue of slender vertical structures, Journal of Structure Engineer - ASCE, 130(7), 1032-1040.

Seyit A. Akdağ \& Ali Dinler. (2009), A new method to estimate Weibull parameters for wind energy applications, Energy Conversion \& Management, 50(7), 1761-1766. 


\section{List of Figure Captions}

Fig. 1. Flowchart of REBMIX

Fig. 2. Results of parameter estimation by REBMIX algorithm for case 1

Fig. 3. Results of parameter estimation by REBMIX algorithm for case 2

Fig. 4. Results of parameter estimation by REBMIX algorithm for case 3

Fig. 5. Results of parameter estimation by REBMIX algorithm for case 4

Fig. 6. Results of parameter estimation by REBMIX algorithm for case 5

Fig. 7. Results of parameter estimation by REBMIX algorithm for case 6

Fig. 8. AIC values with different numbers of bins

Fig. 9. AIC values of models estimated by REBMIX and EM algorithm

Fig. 10. Optimal models by REBMIX and EM

Fig. 11. Computing time by REBMIX and EM

Fig. 12. AIC values with different numbers of bins

Fig. 13. AIC values of models estimated by REBMIX algorithm

Fig. 14. Optimal model by REBMIX algorithm

Fig. 15. Optimal model of wind speed and circular variable

Fig. 16. Optimal model by EM algorithm-based angular-linear modeling approach 


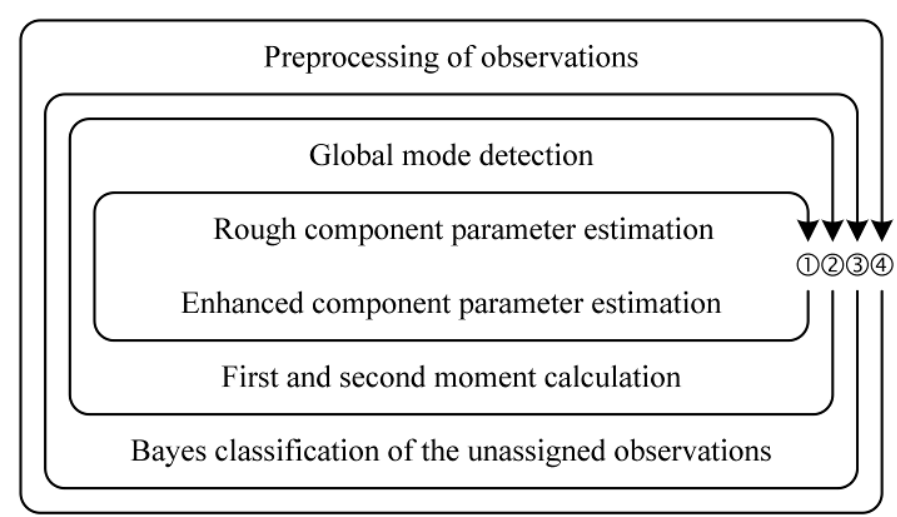

Fig. 1. Flowchart of REBMIX 

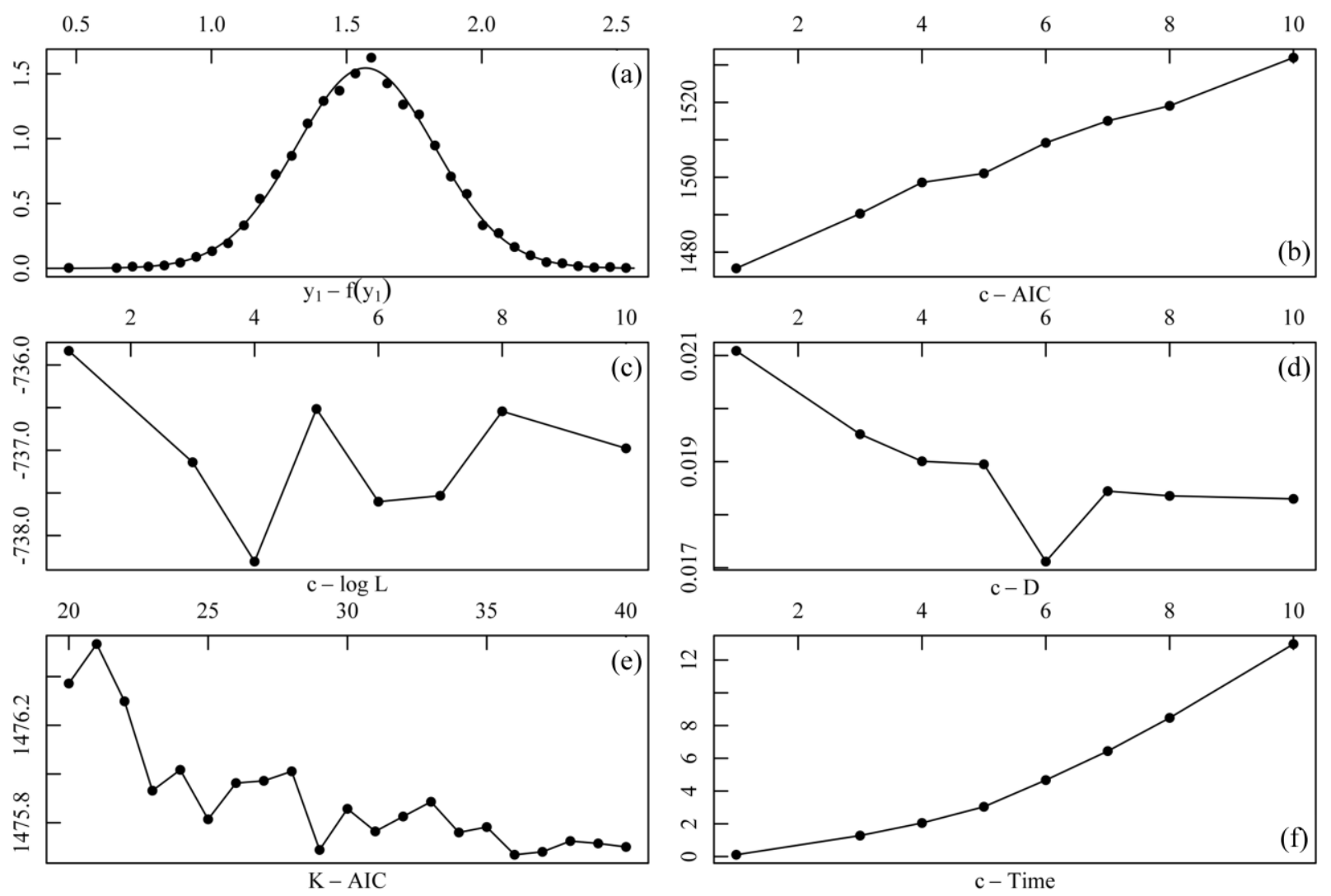

Fig. 2. Results of parameter estimation by REBMIX algorithm for case 1 

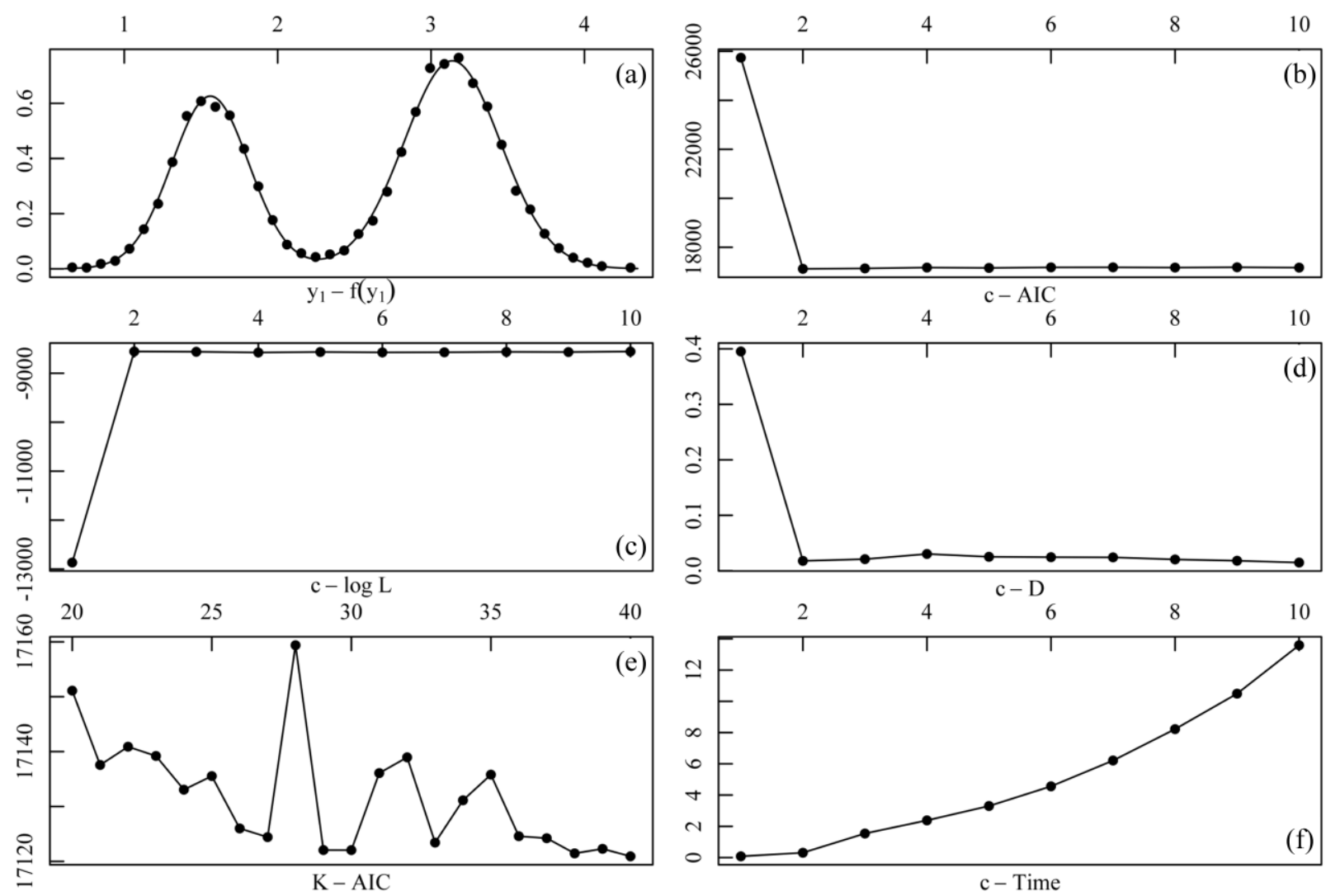

Fig. 3. Results of parameter estimation by REBMIX algorithm for case 2 

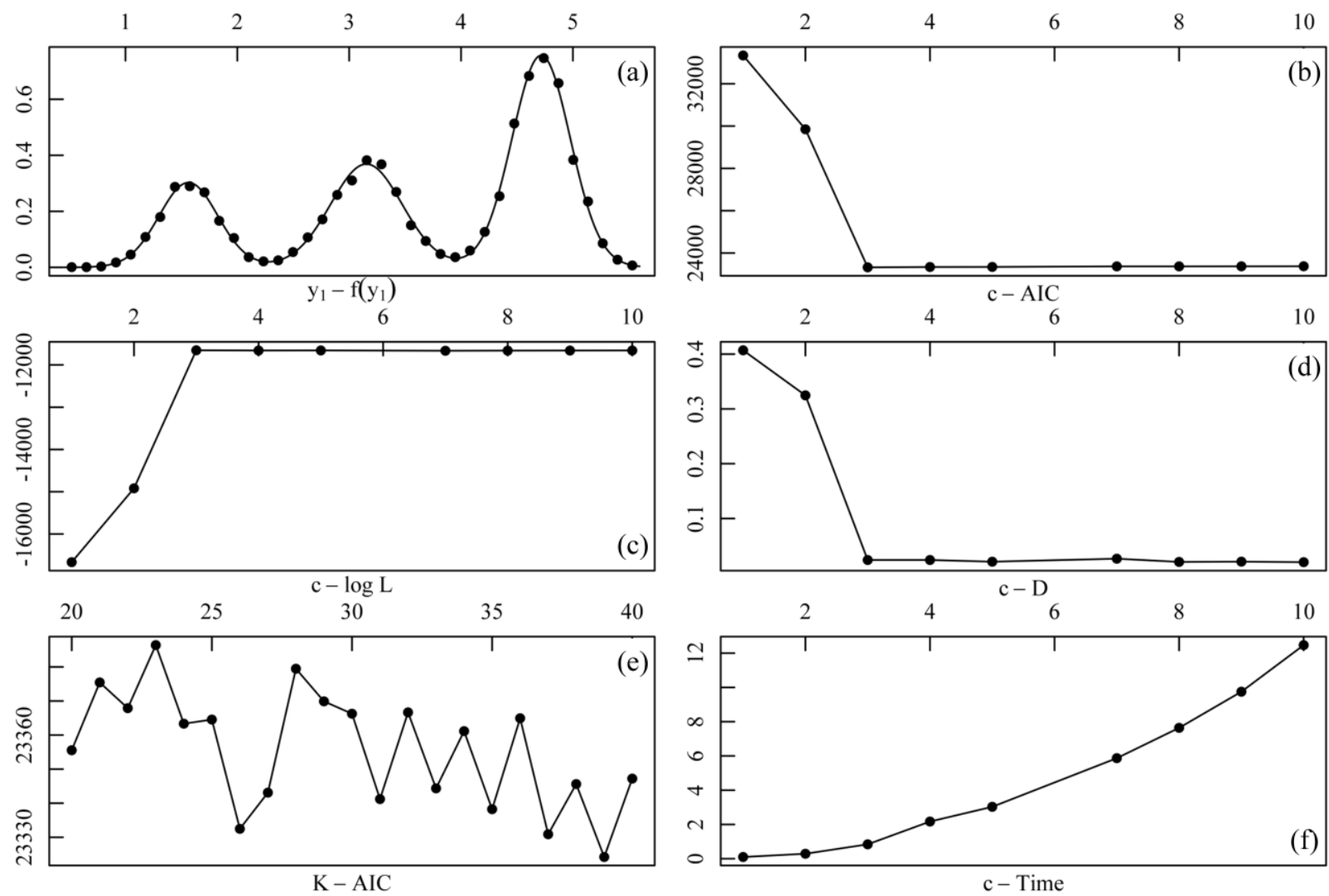

Fig. 4. Results of parameter estimation by REBMIX algorithm for case 3 

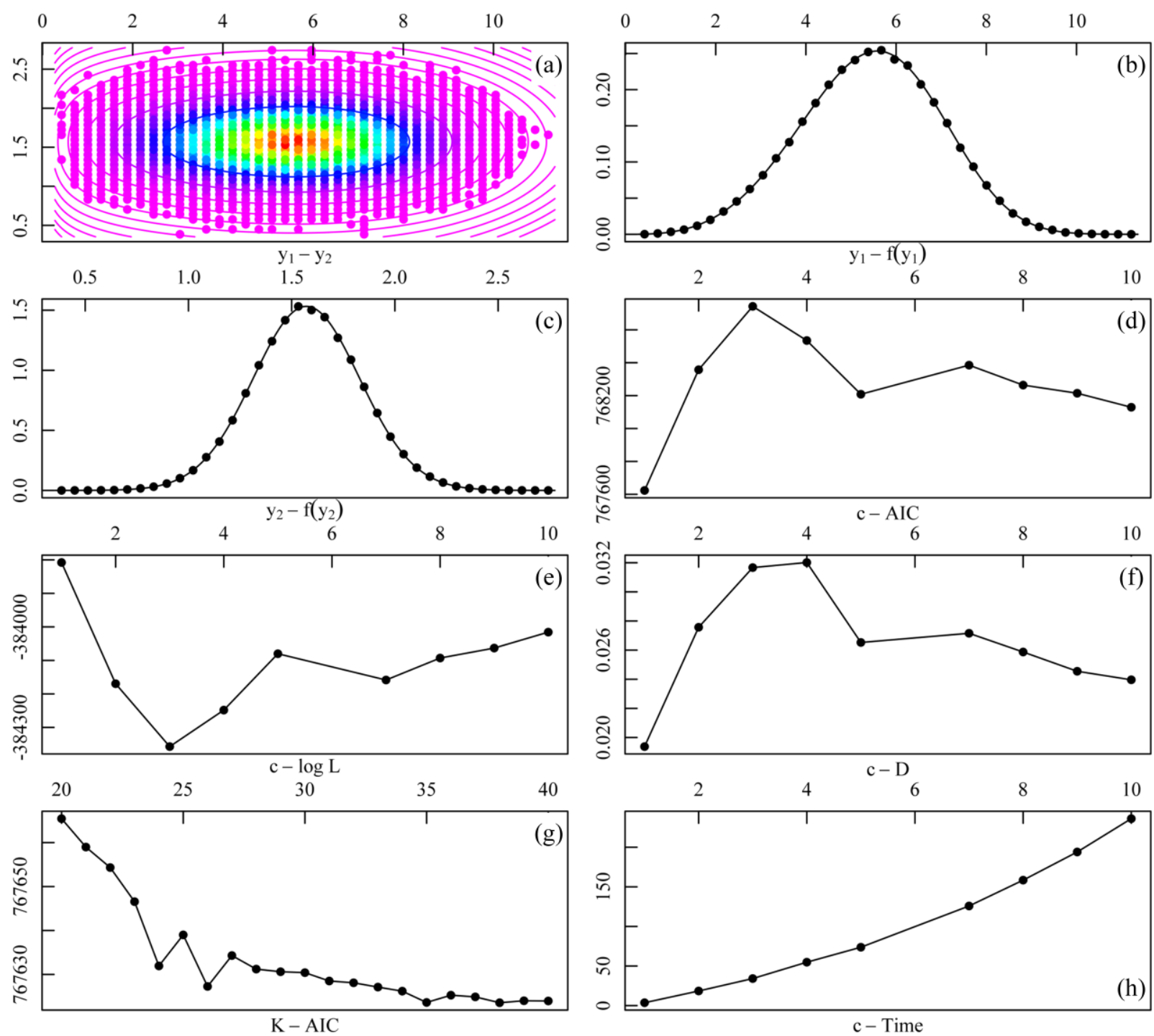

Fig. 5. Results of parameter estimation by REBMIX algorithm for case 4 

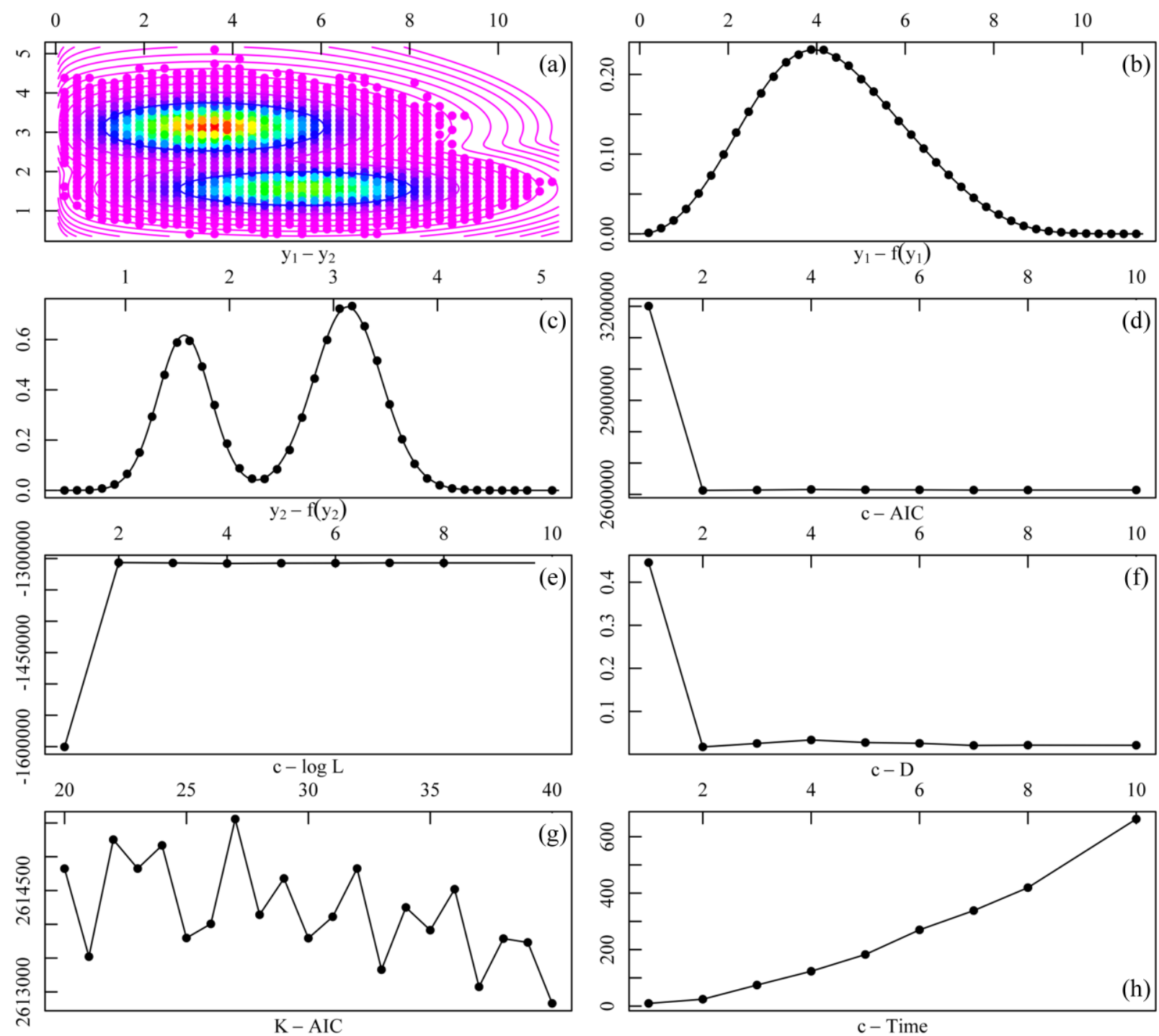

Fig. 6. Results of parameter estimation by REBMIX algorithm for case 5 

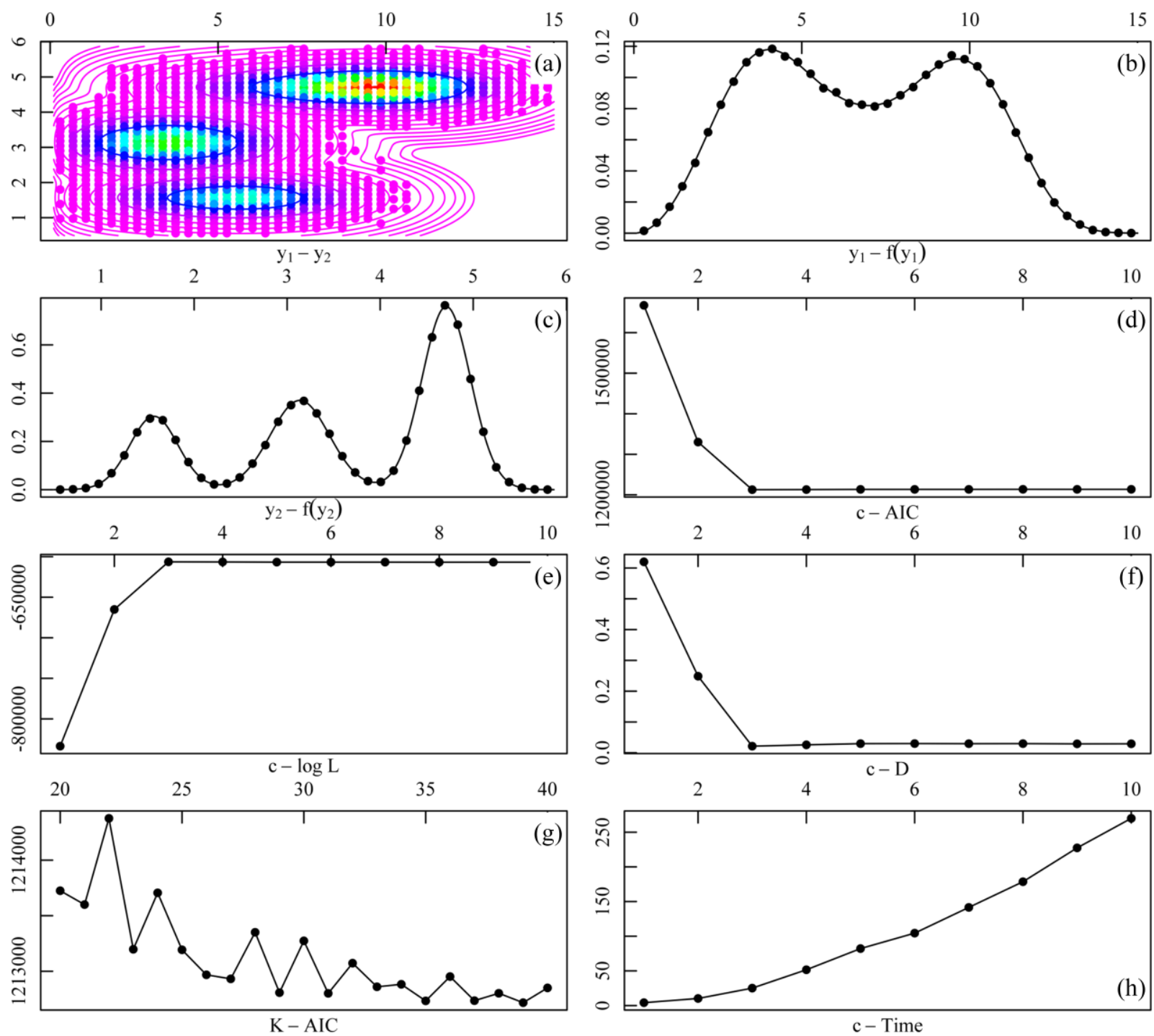

Fig. 7. Results of parameter estimation by REBMIX algorithm for case 6 


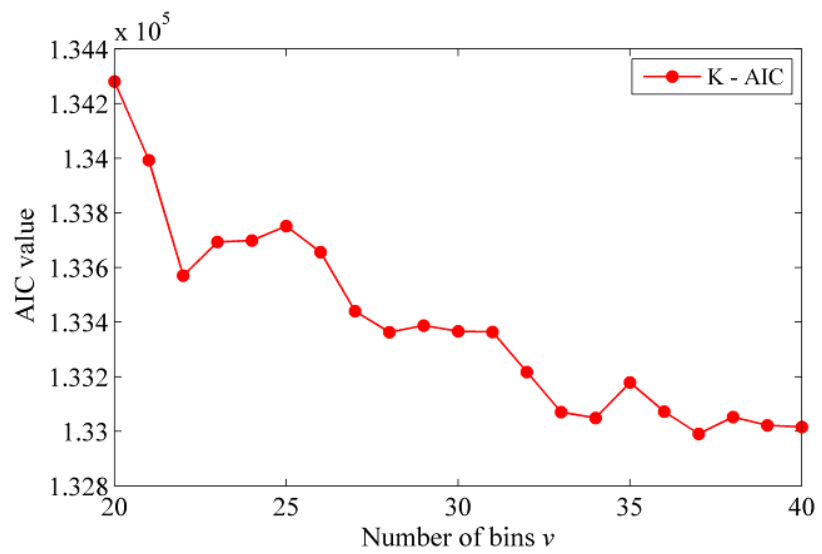

Fig. 8. AIC values with different numbers of bins 


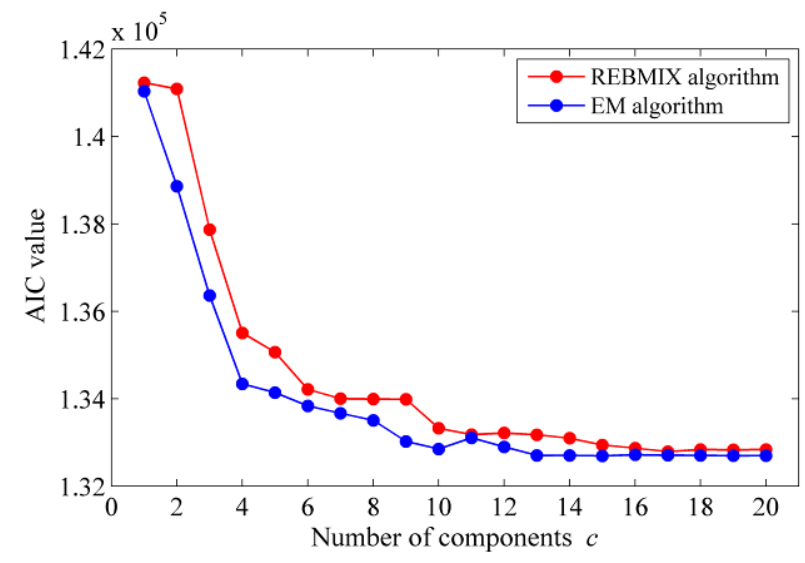

Fig. 9. AIC values of models estimated by REBMIX and EM algorithm 


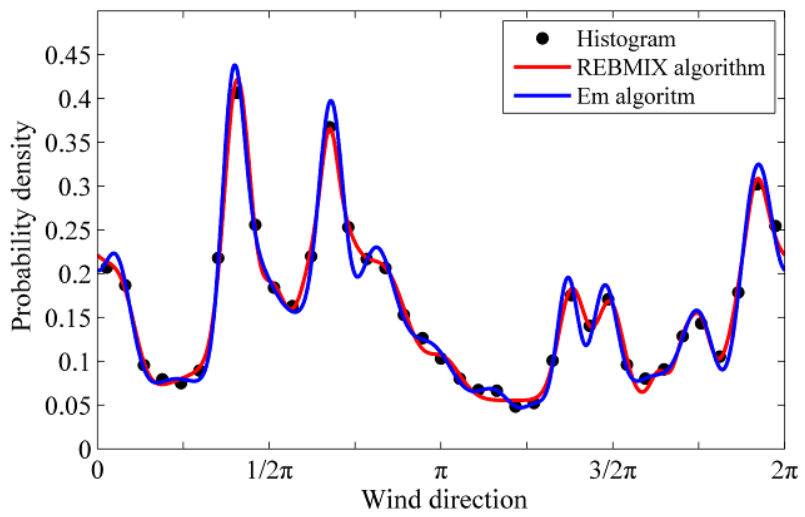

Fig. 10. Optimal models by REBMIX and EM 


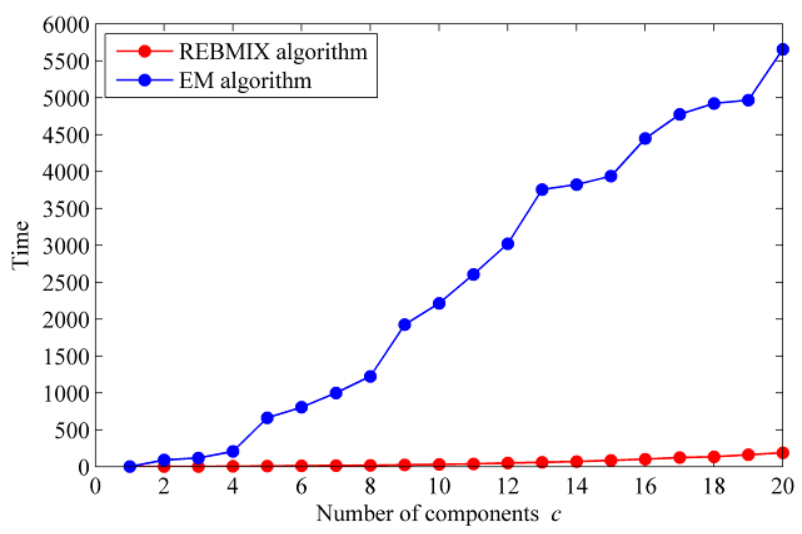

Fig. 11. Computing time by REBMIX and EM 


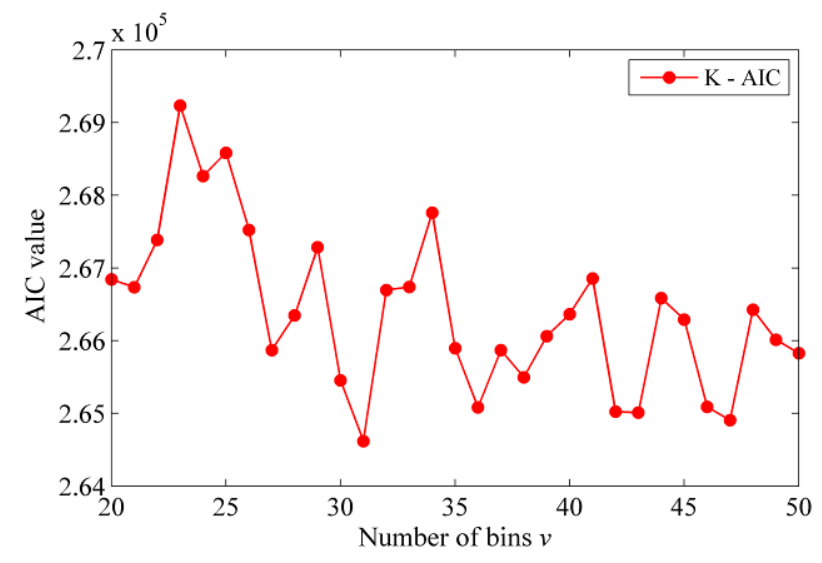

Fig. 12. AIC values with different numbers of bins 


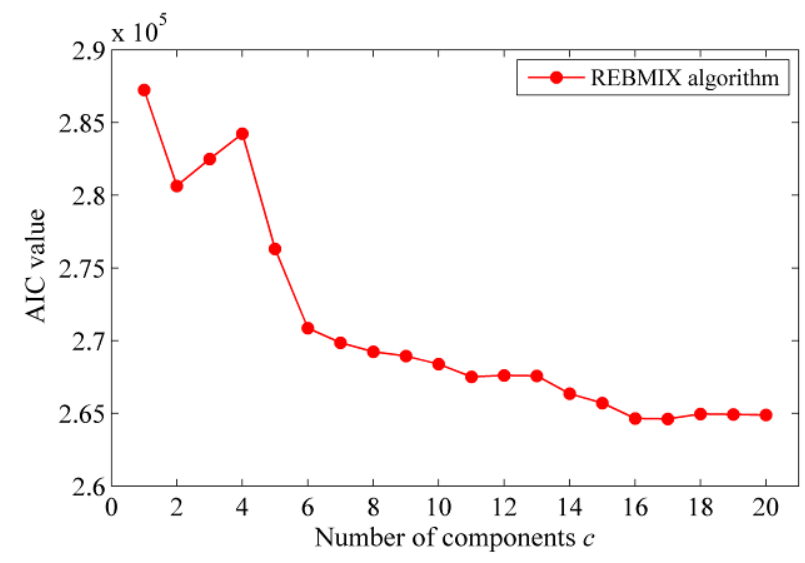

Fig. 13. AIC values of models estimated by REBMIX algorithm 
Wind speed

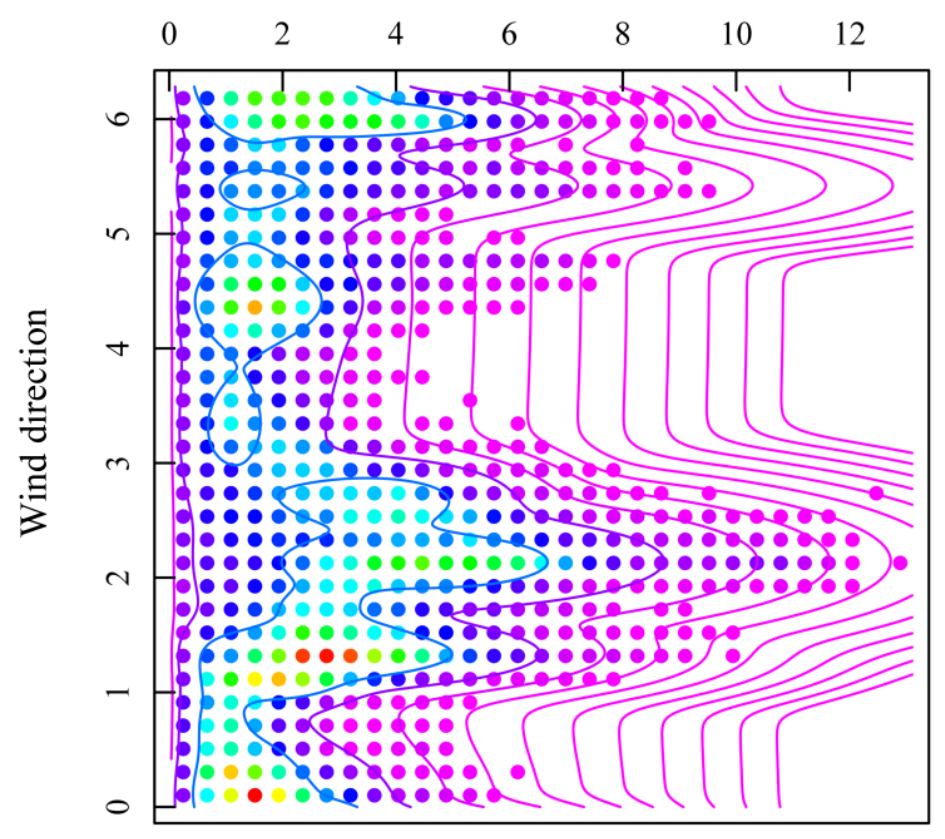

Fig. 14. Optimal model by REBMIX algorithm 


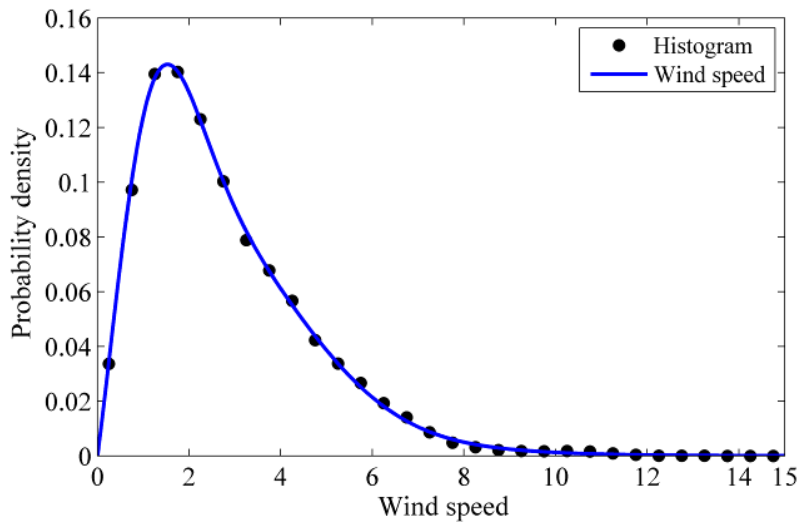

(a) Model of wind speed

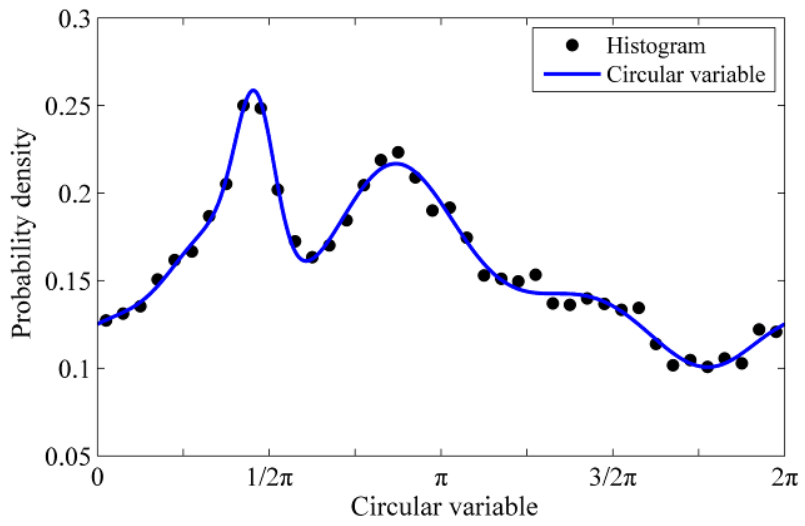

(b) Model of circular variable

Fig. 15. Optimal model of wind speed and circular variable 


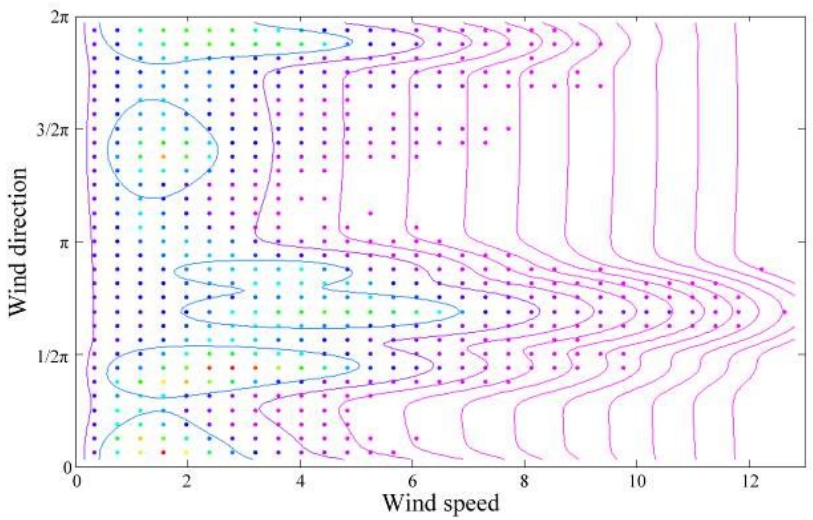

Fig. 16. Optimal model by EM algorithm-based angular-linear modeling approach 


\section{List of Table Caption}

Table 1. True values and predictive values of parameters in example 1

Table 2. True values and predictive values of parameters in example 2 
Table 1. True values and predictive values of parameters in example 1

\begin{tabular}{|c|c|c|c|c|c|c|c|c|c|c|}
\hline \multirow{3}{*}{ Case } & \multirow{3}{*}{$c$} & \multicolumn{3}{|c|}{ True value } & \multicolumn{6}{|c|}{ Predictive value } \\
\hline & & \multirow{2}{*}{$w$} & \multirow{2}{*}{$\mu$} & \multirow{2}{*}{$\kappa$} & \multicolumn{2}{|c|}{$w$} & \multicolumn{2}{|c|}{$\mu$} & \multicolumn{2}{|c|}{$\kappa$} \\
\hline & & & & & value & error & value & error & value & error \\
\hline 1 & 1 & 1 & $\pi / 2$ & 15 & 1.00 & $0 \%$ & 1.56 & $0.06 \%$ & 15.24 & $1.64 \%$ \\
\hline \multirow{2}{*}{2} & \multirow{2}{*}{2} & 0.4 & $\pi / 2$ & 15 & 0.39 & $0.47 \%$ & 1.56 & $0.62 \%$ & 15.78 & $5.22 \%$ \\
\hline & & 0.6 & $\pi$ & 10 & 0.61 & $0.31 \%$ & 3.13 & $0.11 \%$ & 10.12 & $1.19 \%$ \\
\hline \multirow{3}{*}{3} & \multirow{3}{*}{3} & 0.2 & $\pi / 2$ & 15 & 0.20 & $0.36 \%$ & 1.56 & $0.48 \%$ & 14.53 & $3.11 \%$ \\
\hline & & 0.3 & $\pi$ & 10 & 0.30 & $0.12 \%$ & 3.15 & $0.38 \%$ & 9.72 & $2.76 \%$ \\
\hline & & 0.5 & $3 \pi / 2$ & 15 & 0.50 & $0.22 \%$ & 4.71 & $0.08 \%$ & 14.69 & $2.03 \%$ \\
\hline
\end{tabular}


Table 2. True values and predictive values of parameters in example 2

\begin{tabular}{|c|c|c|c|c|c|c|c|c|c|c|c|}
\hline \multirow{2}{*}{ Case } & \multirow{2}{*}{$c$} & \multicolumn{10}{|c|}{ True value } \\
\hline & & \multicolumn{2}{|c|}{$w$} & \multicolumn{2}{|c|}{$\alpha$} & \multicolumn{2}{|c|}{$\beta$} & \multicolumn{2}{|c|}{$\mu$} & \multicolumn{2}{|c|}{$\kappa$} \\
\hline 4 & 1 & \multicolumn{2}{|c|}{1} & \multicolumn{2}{|c|}{6} & \multicolumn{2}{|c|}{4} & \multicolumn{2}{|c|}{$\pi / 2$} & \multicolumn{2}{|c|}{15} \\
\hline \multirow{2}{*}{5} & \multirow{2}{*}{2} & \multicolumn{2}{|c|}{0.4} & \multicolumn{2}{|c|}{6} & \multicolumn{2}{|c|}{4} & \multicolumn{2}{|c|}{$\pi / 2$} & \multicolumn{2}{|c|}{15} \\
\hline & & \multicolumn{2}{|c|}{0.6} & \multicolumn{2}{|c|}{4} & \multicolumn{2}{|c|}{3} & \multicolumn{2}{|c|}{$\pi$} & \multicolumn{2}{|c|}{10} \\
\hline \multirow{3}{*}{6} & \multirow{3}{*}{3} & \multicolumn{2}{|c|}{0.2} & \multicolumn{2}{|c|}{6} & \multicolumn{2}{|c|}{6} & \multicolumn{2}{|c|}{$\pi / 2$} & \multicolumn{2}{|c|}{15} \\
\hline & & \multicolumn{2}{|c|}{0.3} & \multicolumn{2}{|c|}{4} & \multicolumn{2}{|c|}{3} & & & & \\
\hline & & & & & & & 6 & & & & \\
\hline & & & & & & Predict & ve value & & & & \\
\hline Case & $c$ & & & & & & $\beta$ & & & & \\
\hline & & value & error & value & error & value & error & value & error & value & error \\
\hline 4 & 1 & 1.0 & $0 \%$ & 6.001 & $0.02 \%$ & 3.993 & $0.18 \%$ & 1.571 & $0.01 \%$ & 15.004 & $0.02 \%$ \\
\hline 5 & & 0.396 & $1.01 \%$ & 6.010 & $0.17 \%$ & 4.026 & $0.65 \%$ & 1.565 & $0.37 \%$ & 15.511 & $3.40 \%$ \\
\hline 5 & 2 & 0.603 & $0.52 \%$ & 4.010 & $0.25 \%$ & 3.002 & $0.07 \%$ & 3.136 & $0.18 \%$ & 9.4368 & $5.63 \%$ \\
\hline & & 0.199 & $0.49 \%$ & 6.018 & $0.31 \%$ & 6.020 & $0.33 \%$ & 1.567 & $0.24 \%$ & 14.906 & $0.63 \%$ \\
\hline 6 & 3 & 0.299 & $0.33 \%$ & 3.997 & $0.08 \%$ & 2.999 & $0.03 \%$ & 3.135 & $0.21 \%$ & 9.8122 & $1.88 \%$ \\
\hline & & 0.500 & $0.02 \%$ & 10.002 & $0.02 \%$ & 5.945 & $0.92 \%$ & 4.710 & $0.05 \%$ & 14.679 & $2.14 \%$ \\
\hline
\end{tabular}

